\title{
OS EFEITOS DA ESTIMULAÇÃO ELÉTRICA FUNCIONAL NA ASSIMETRIA CORTICAL INTER-HEMISFÉRICA
}

\author{
Letícia Ecard', Andressa Pitanga S. da Silva², Marijose Peçanha Neto ${ }^{3}$, \\ Heloisa Veiga ${ }^{4}$, Maurício Cagy $^{5}$, Roberto Piedade ${ }^{6}$, Pedro Ribeiro $^{7}$
}

\begin{abstract}
RESUMO - O objetivo do presente estudo foi avaliar os efeitos da estimulação elétrica funcional na assimetria cortical inter-hemisférica. Para tal, simultaneamente ao registro da atividade eletroencefalográfica, realizou-se eletroestimulação no antebraço direito para estimulação da extensão do indicador. A amostra consistiu de 45 sujeitos randomizados em 3 grupos de 15 sujeitos cada: grupo controle (submetido a 24 blocos de estimulação com intensidade de corrente zero), grupo 1 (24 blocos) e grupo 2 (36 blocos). A assimetria entre os pares de eletrodos F3-F4, C3-C4 e P3-P4 foi analisada ao longo dos grupos através de uma Anova. Os resultados apontaram para uma interação grupo x eletrodo e uma tendência de diminuição da assimetria inter-hemisférica após a eletroestimulação.
\end{abstract}

PALAVRAS-CHAVE: FES, EEG, EEGq, assimetria Inter-hemisférica.

\begin{abstract}
The effects of functional electrical stimulation on cortical interhemispheric asymmetry
ABSTRACT - The aim of the present study was to assess the effects of functional electrical stimulation (FES) on cortical interhemispheric asymmetry. Electrostimulation was performed on the right forearm to stimulate the extension of the index finger. EEG activity was recorded simultaneously. The sample included 45 subjects randomly divided into 3 groups of 15 subjects each: control group (submitted to 24 blocks of stimulation at a null intensity current), group 1 (24 blocks) and group 2 (36 blocks). Interhemispheric asymmetry between F3-F4, C3-C4 and P3-P4 was analyzed through an Anova. Results pointed out to a group $\mathrm{x}$ electrode interaction and a general tendency of asymmetry decrease after stimulation.
\end{abstract}

KEY WORDS: FES, EEG, EEGq, interhemispheric asymmetry.

Os estudos na área do comportamento motor têm evoluído ao longo dos tempos e novos padrões têm surgido com o objetivo de ampliar o entendimento sobre as interações neuromusculares na produção de uma ação motora ${ }^{1-3}$. Existem inúmeras possibilidades de aplicação do aprendizado motor no processo de reabilitação motora-funcional. A partir de tais argumentos, torna-se essencial o entendimento de como se processa esse aprendizado, quer seja em condições normais, bem como em circunstâncias patológicas, para que se desenvolvam estratégias eficazes de tratamento. Com relação às técnicas que são utilizadas a fim de gerar a reabilitação funcional daqueles que necessitam de cuidados, pode-se citar a estimulação elétrica funcional (FES) como um importante aliado para a potencialização terapêutica ${ }^{4}$; entretanto, os efeitos de sua aplicação na dinâmica cerebral são ainda muito pouco explorados. Outra técnica utilizada é o exame eletrencefalográfico (EEG), que permite mapear não só a região cerebral ativada durante a execução de uma tarefa, como também quantificar o nível de atividade elétrica neuronal nestas regiões. Através de métodos de análises quantitativas (EEGq) torna-se possível, por exemplo, observar assimetrias inter e intra-hemisféricas geradas durante o aprendizado de uma tarefa motora ${ }^{5-6}$.

\footnotetext{
${ }^{1}$ Mestranda, Laboratório de Mapeamento Cerebral e Integração Sensório-Motora, Instituto de Psiquiatria (IPUB), Universidade Federal do Rio de Janeiro, Rio de Janeiro RJ, Brasil (UFRJ); ${ }^{2}$ Mestre em Ciência da Motricidade Humana, Universidade Castelo Branco, Rio de Janeiro RJ, Brasil (PROCIMH-UCB), Professora, Universidade Vale do Rio Doce - UNIVALE; ${ }^{3}$ Mestranda em Ciência da Motricidade Humana, Universidade Castelo Branco (PROCIMH-UCB); ${ }^{4}$ Mestre, Laboratório de Mapeamento Cerebral e Integração SensórioMotora (IPUB-UFRJ); ${ }^{5}$ D.Sc. em Engenharia Biomédica, COPPE, UFRJ; ${ }^{6}$ Professor Adjunto III Doutor, Coordenador do Laboratório de Mapeamento Cerebral e Integração Sensório-Motora (IPUB - UFRJ); ${ }^{7}$ Professor Adjunto II PhD, Escola de Educação Física e Desportos (EEFD), Laboratório de Mapeamento Cerebral e Integração Sensório-Motora (IPUB-UFRJ), Professor Pesquisador PROCIMH-UCB.
}

Recebido 16 Janeiro 2007, recebido na forma final 1 Março 2007. Aceito 20 Abril 2007.

Dra. Letícia Ecard - Instituto de Psiquiatria (IPUB) / Laboratório de Mapeamento Cerebral e Integração Sensório-Motora / Universidade Federal do Rio de Janeiro - Avenida Venceslau Brás 71 / Fundos - 22290-140 Rio de Janeiro RJ - Brasil. E-mail: leticiaecard@bol.com.br 
Neste sentido, a investigação de padrões neurais ligados a processos sensoriais, motores e cognitivos, pode ser realizada através da monitoração da atividade cortical ${ }^{7}$, contribuindo dessa maneira para que mecanismos relacionados à integração sensório-motora sejam explicados. Porém, ainda são poucos os estudos existentes na literatura que abordam os efeitos da eletroestimulação na dinâmica cortical e na atividade eletrencefalográfica.

Neste contexto, na busca de uma compreensão mais detalhada sobre os efeitos desencadeados pelo FES, a presente investigação teve como objetivo observar alterações na assimetria inter-hemisférica produzidas pela estimulação elétrica funcional.

\section{MÉTODO}

Amostra - A amostra do estudo consistiu de 45 sujeitos voluntários, sendo 21 do sexo feminino e 24 do sexo masculino, entre 20 e 40 anos de idade. Os sujeitos foram divididos aleatoriamente em três grupos: grupo controle, grupo 1 e grupo 2 . Foram incluídos no estudo apenas sujeitos sadios, sem nenhum comprometimento cognitivo e que não estivessem fazendo uso de substâncias psicotrópicas ou psicoativas. Assim, o inventário de Edinburgh ${ }^{8}$ foi aplicado e somente os sujeitos destros foram incluídos no estudo. Todos os sujeitos assinaram um termo de consentimento livre e esclarecido, no qual foram detalhadas as condições experimentais. O experimento foi submetido e aprovado pelo Comitê de Ética do Instituto de Psiquiatria.

Procedimento experimental - A sala utilizada para a realização do exame foi preparada com um isolamento de som e, durante o exame, as luzes foram reduzidas ao máximo. Os sujeitos sentaram-se confortavelmente em uma cadeira, tendo seus braços apoiados em uma mesa colocada a sua frente, com o objetivo de diminuir os artefatos musculares. Foi colocada uma venda nos olhos dos sujeitos para evitar possíveis estímulos visuais e para diminuir os artefatos de piscada de olhos. Foi utilizado um aparelho de estimulação elétrica funcional da marca Ibramed, para estimular a extensão do dedo indicador da mão direita. Essa mesma mão ficou apoiada em um suporte posicionado em cima da mesa, a qual possuía velcros para isolar ou imobilizar todos os outros dedos da mão, deixando livre apenas o dedo indicador para a estimulação (Fig 1).

O aparelho de estimulação elétrica funcional foi calibrado para esse experimento pelo osciloscópio Tektronix TDS 220, Digital-Real-Time de $100 \mathrm{MHz}$ (megahertz), e seguiu os seguintes parâmetros: freqüência da corrente $(R): 48,8 \mathrm{~Hz}$; intensidade da corrente (A): $2.10^{-3} \mathrm{~A}$; tempo de passagem da corrente (tempo on): 4,86 s; tempo de ausência de passagem da corrente (tempo off): $8,39 \mathrm{~s}$; período da corrente (T) em on: $320 \mu \mathrm{s}$; período da corrente (T) em off: $25 \mu \mathrm{s}$; área do eletrodo: $4,5.10^{-4} \mathrm{~m}^{2}$; densidade da corrente: $44 \mathrm{~A} / \mathrm{m}^{2}$.

A impedância variou entre 800 e 1500 . Para atingir essa faixa de variação, o local da pele onde foram posicionados os eletrodos para a passagem da corrente teve seus

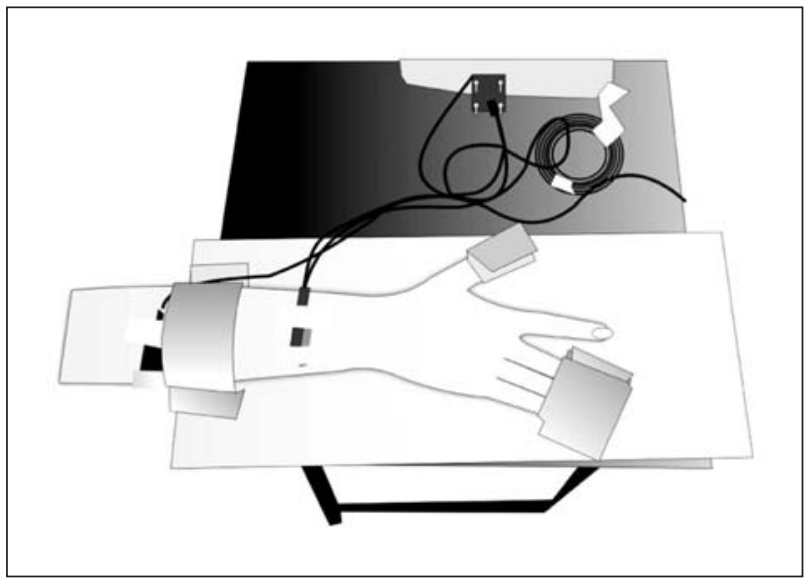

Fig 1. Suporte para posicionamento de antebraço e mão.

pêlos raspados com lâmina de barbear e limpo com álcool. Além disso, foi usado um gel entre o eletrodo e a pele do sujeito para facilitar a condução da corrente. Esses eletrodos foram fixados um a $5 \mathrm{~cm}$ do epicôndilo lateral na face lateral do antebraço e o outro a $12 \mathrm{~cm}$ do primeiro, ocupando a face posterior do antebraço, seguindo a trajetória do tendão do extensor do dedo indicador. O experimento foi realizado com trilhas e blocos. Cada trilha foi estabelecida como 1 tempo de estimulação, chamado tempo on com 4,86 s de passagem de corrente, mais 1 tempo de repouso, chamado tempo off com 8,39s sem passagem de corrente. Cada bloco foi constituído de 6 trilhas. O grupo controle realizou 24 blocos, com uma pausa de $1 \mathrm{~min}$ a cada 6 blocos realizados. Porém, a intensidade da corrente desse grupo foi de zero A. O grupo 1 realizou 24 blocos com pausa de $1 \mathrm{~min}$. a cada 6 blocos, obedecendo integralmente às condições do aparelho descritas anteriormente. O grupo 2 realizou 36 blocos com pausa de 1 min a cada 6 blocos, nas mesmas condições do grupo 1, diferindo deste apenas em números de blocos realizados. Simultaneamente à estimulação elétrica funcional do músculo extensor dos dedos, foi realizada a captação do sinal eletroencefalográfico.

Aquisição de dados - Para a captação do sinal eletrencefalográfico foi utilizado o aparelho Braintech 3000 (EMSA Instrumentos Médicos, Brasil), sistema que utiliza uma placa conversora analógica-digital (A/D) de 32 canais com resolução de12 bits, colocada em um slot ISA de um Pentium III - com processador de $750 \mathrm{MHz}$. Sinais eletrofisiológicos foram filtrados analogicamente entre $0,16 \mathrm{~Hz}$ (passa-altas) e $35 \mathrm{~Hz}$ (passa-baixas), tendo uma taxa de amostragem de $200 \mathrm{~Hz}$. Foi utilizado o software de aquisição denominado EEG Captação (Emsa-DELPHI 5.0), com um filtro notch de $60 \mathrm{~Hz}$, e ainda filtros de corte de $0,3 \mathrm{~Hz}$ (passa-baixas) e 25 $\mathrm{Hz}$ (passa-altas). O sistema internacional $10-20^{9}$ foi usado para a colocação de 20 eletrodos monopolares ao longo do escalpo (áreas: frontal, temporal, central, parietal e occipital) e um eletrodo em cada orelha (lóbulo). Os eletrodos foram montados em uma touca de nylon (ElectroCap Inc., Fairfax, VA, USA) com sistema 10-20 pré-fixados. Os lóbulos das orelhas foram usados como referências (bi-auricular). Foram verificados, a priori, os níveis de impedância de cada 
eletrodo, cujos valores foram mantidos entre 5-10 K $\Omega$. Para que os sinais adquiridos tivessem amplitude total (pico a pico) menor que $100 \mu \mathrm{V}$, ele foi amplificado com ganho de aproximadamente 20000 vezes. A atividade elétrica ocular foi estimada com a colocação de dois eletrodos de $9 \mathrm{~mm}$ de diâmetro montados de forma bipolar. Os eletrodos foram posicionados, respectivamente, acima e abaixo da órbita do olho direito, para registrar os movimentos oculares verticais e, no canto externo do mesmo, para registrar movimentos oculares horizontais. Um programa de visualização, denominado EEG Telas (Emsa-Delphi 5.0) foi utilizado para eliminar os artefatos de movimento ocular.

Análise de dados e cálculo da variável dependente Após a coleta de dados e seu respectivo arquivamento, foi realizado o cálculo da variável dependente extraída da eletrencefalografia quantitativa (EEGq). Uma rotina em Matlab $5.3^{\circledR}$ (Mathworks Inc., Naticj, MA, USA) foi implementada para realizar a análise espectral e estimar o parâmetro específico de interesse: assimetria inter-hemisférica na banda beta (13-25 Hz).

A assimetria reflete uma distribuição não-balanceada de energia (potência) entre pares de eletrodos homólogos (i.e., eletrodos em uma mesma posição, mas em lados opostos no escalpo). Especificamente, a assimetria é entendida como um gradiente de energia existente entre um par de eletrodos. Desta forma, o gradiente de inclinação se concentra no eletrodo com maior quantidade de energia. Índices computacionais de assimetria possibilitam a comparação entre áreas corticais intra e inter-hemisféricas e, consequentemente, a detecção do equilíbrio de atividade entre áreas corticais distintas ${ }^{10}$. $\mathrm{O}$ cálculo matemático da assimetria é expresso através da equação: \% assimetria = $(E-D / E+D) \times 100$, onde $E$ refere-se ao eletrodo esquerdo do par homólogo e $\mathrm{D}$ ao direito.

Cabe ressaltar que o cálculo da variável dependente foi realizado levando em consideração apenas o momento pós-estimulação.
Banda de freqüência e localização espacial - Os dados eletrencefalográficos referem-se exclusivamente à banda beta $(13-35 \mathrm{~Hz})$. A escolha por esta banda se justifica pelo fato de beta estar particularmente associada a processos de integração sensório-motora ${ }^{11}$. O sistema 10-20 é sempre utilizado como um todo, porém, os eletrodos localizados em áreas frontais, centrais e parietais foram selecionados por estarem de acordo com os objetivos do projeto. As áreas frontais foram analisadas devido à sua associação com mecanismos de atenção, motivação e planejamento. Eletrodos centrais e parietais foram analisados por serem representativos de áreas pré-motoras e motoras primárias, além de áreas somatosensoriais primárias ${ }^{12}$.

Análise estatística - Para a análise da assimetria foi realizada uma Anova two-way, grupo x eletrodo (3 x 3). Um teste Post Hoc (Scheffé) foi empregado a posteriori. As condições experimentais foram estabelecidas como: grupo controle (C), grupo 1 (24) e grupo 2 (36), em função do número de estimulações recebidas; os pares de eletrodos selecionados para a análise foram: F3-F4, C3-C4 e P3-P4. Como dito anteriormente, apenas a banda beta e o momento pós-estimulação foram analisados.

\section{RESULTADOS}

A Figura 2 ilustra a variação dos valores de assimetria ao longo das condições experimentais (grupos) e dos eletrodos pré-estabelecidos. A Anova não revelou efeitos principais para grupo $(p=0,315)$ ou eletrodo $(p=0,167)$. Entretanto, foi observada uma interação grupo x eletrodo $(p=0,014)$. Especificamente, observou-se uma tendência de diminuição da assimetria ao longo dos grupos e eletrodos. Neste contexto, a Figura 3 ilustra a dinâmica da distribuição de potência absoluta nos eletrodos analisados. A tendência à diminuição da assimetria é caracterizada por maior

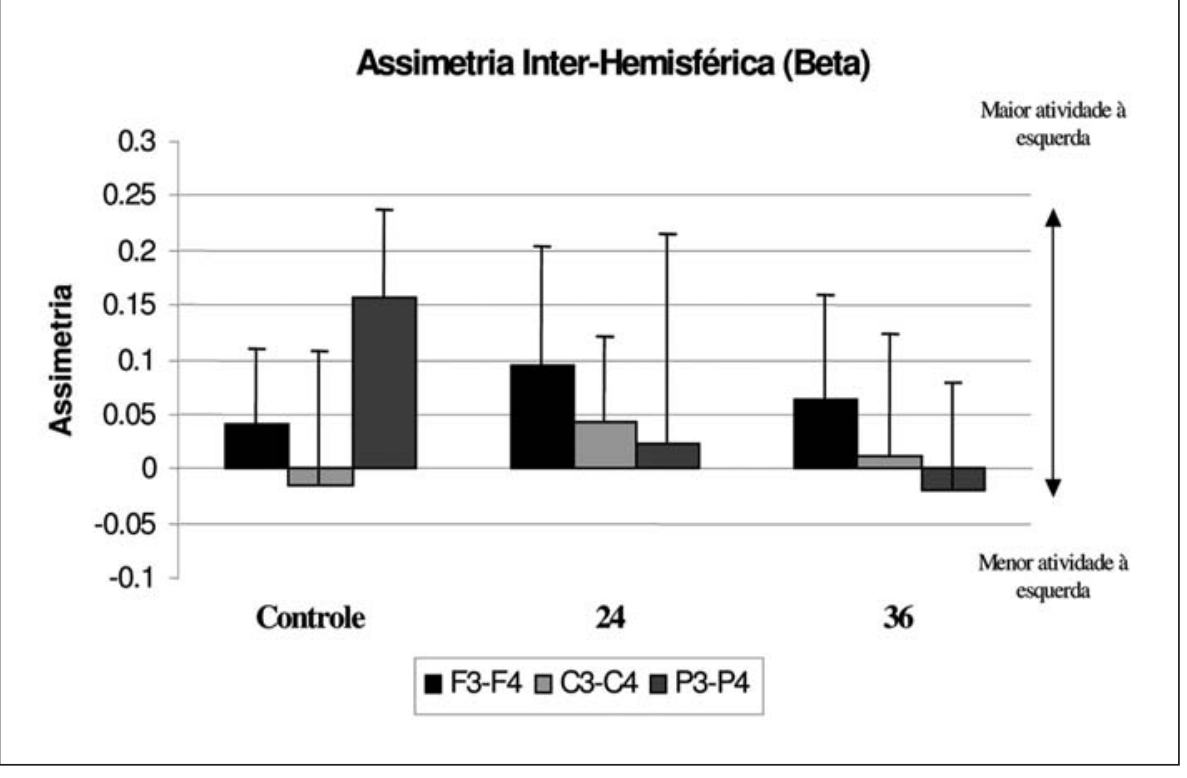

Fig 2. Variação dos valores de assimetria ao longo dos grupos experimentais nos pares de eletrodos $F 3-$ F4, C3-C4 e P3-P4 $(p \leq 0,05)$. 


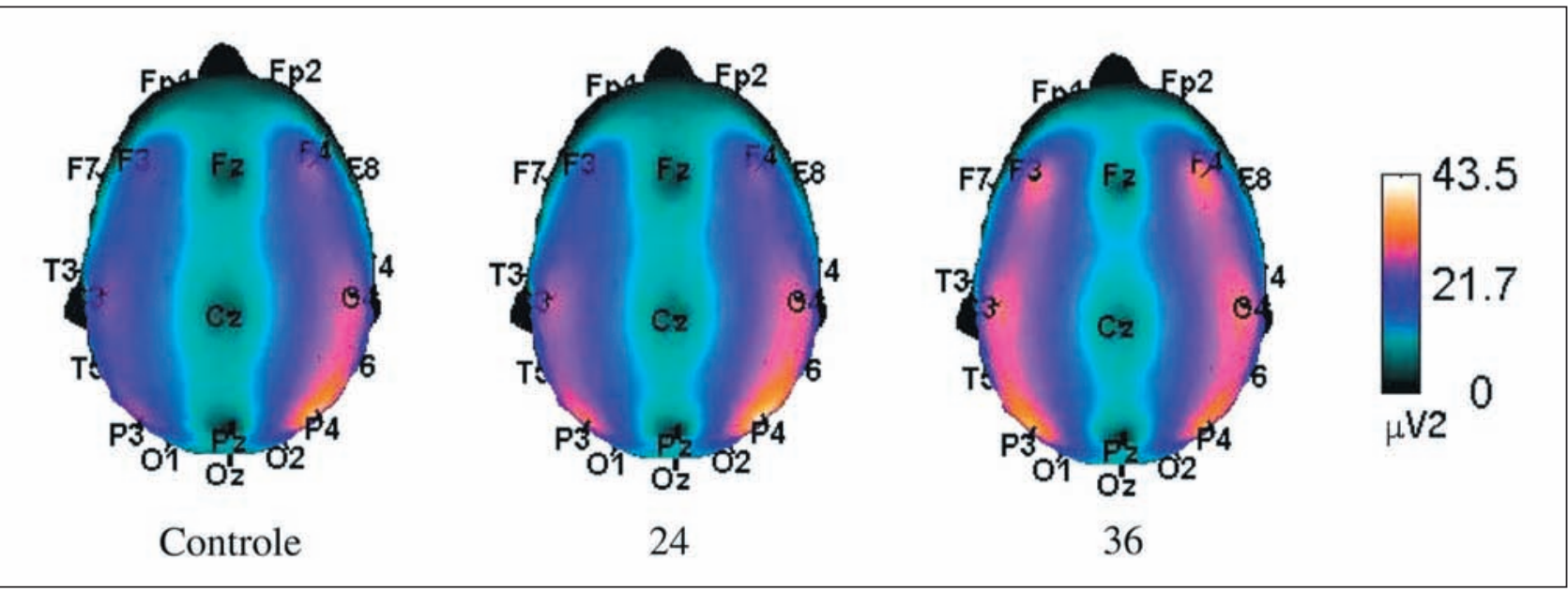

Fig 3. Mapas corticais da distribuição de potência absoluta nos grupos experimentais.

balanceamento de energia (potência) ao longo dos diferentes grupos experimentais.

\section{DISCUSSÃO}

Apesar da comprovada relevância clínica e experimental tanto de análises eletrencefalográficas quanto da estimulação elétrica, estudos relacionando a eletroestimulação com alterações corticais (expressas por variáveis do $\mathrm{EEGq)}$ ) são praticamente inexistentes na literatura. Neste contexto, o presente estudo se propôs a analisar as variações na assimetria inter-hemisférica decorrentes da estimulação elétrica funcional. Os resultados da análise estatística não evidenciaram efeitos principais para as variáveis independentes (grupo e eletrodo). Entretanto, a ausência de efeitos principais significativos pode estar relacionada a fatores tais como: a variabilidade inter-sujeitos e o estado fisiológico dos mesmos na hora do exame. Por outro lado, foi observada uma interação estatisticamente significativa, grupo $x$ eletrodo, caracterizada por uma tendência de diminuição da assimetria. Cabe ressaltar que, estatisticamente, a existência de uma interação sobrepõe qualquer efeito principal. Em outras palavras, interações fornecem uma idéia mais fidedigna do fenômeno observado. Assim, os resultados do presente estudo indicam que a interação entre a quantidade de eletroestimulação aplicada e a área cortical analisada é o que explica as mudanças observadas no perfil eletrencefalográfico dos sujeitos.

Diversos estudos têm empregado análises de assimetria sob diversos paradigmas experimentais. Sabese que uma assimetria evidente e persistente entre áreas homólogas do cérebro constitui sempre uma anormalidade, em qualquer idade. Assim, medidas de assimetria têm sido utilizadas na avaliação de pato- logias distintas, como depressão maior ${ }^{13}$, dislexia ${ }^{14,15}$, transtorno do pânico ${ }^{16}$ e como um índice do risco para o desenvolvimento de ansiedade ${ }^{17}$. Mais recentemente, análises de assimetria têm sido empregadas no entendimento da dinâmica da integração sensóriomotora. Pfurtscheller et al. ${ }^{18}$ estudaram processos assimétricos no córtex frontal e seus possíveis correlatos com o planejamento e preparação do gesto motor. Outros estudos investigaram assimetrias corticais durante o controle de movimentos manuais e bi-manuais $^{19}$, performance em idosos ${ }^{20}$ e em tarefas sensóriomotoras simultâneas a atividades neuropsicológicas ${ }^{21}$. Entretanto, como citado anteriormente, praticamente não há relatos na literatura de estudos relacionando medidas de assimetria à eletroestimulação.

Um dos poucos estudos que combinaram eletroestimulação e atividade cortical foi realizado por Tecchio et al. ${ }^{22}$. Dezoito pacientes que haviam se recuperado parcialmente ou totalmente de disfunções decorrentes de um acidente vascular cerebral isquêmico (infarto mono-hemisférico na região da artéria cerebral média) foram submetidos a um exame de magnetoencefalografia em repouso e durante estimulação elétrica do nervo mediano contralateral. 0 exame foi realizado na fase aguda (T0: média de 5 dias após o ataque isquêmico) e pós-aguda (T1: média de 6 meses). Os resultados indicaram uma redução significativa de todas as assimetrias em T1 quando comparadas a TO. Os autores argumentam que uma normalização da atividade neural (refletida por diminuição na assimetria e, portanto, maior balanceamento de energia entre os hemisférios) estaria associada a melhor recuperação clínica.

Sabe-se que a recuperação de funções motoras e sensoriais após diferentes lesões no sistema nervo- 
so central (SNC) pode ocorrer ao longo de semanas ou até meses e que a simetria cortical é modificada quando há uma lesão no SNC ${ }^{23-25}$. Neste contexto, a compreensão dos mecanismos subjacentes à recuperação clínica e a relação entre o processo de reabilitação funcional e esta variável eletrencefalográfica se torna fundamental para o desenvolvimento de metodologias que lancem mão integralmente do potencial adaptativo do cérebro. Diversos estudos têm relatado que a reorganização neuronal que ocorre após uma lesão pode ter seus efeitos funcionais aumentados através de certas intervenções, tais como a prática motora, estimulação somatossensorial e agentes farmacológicos ${ }^{26-28}$. Especificamente, experimentos utilizando técnicas de imagem têm demonstrado que a redução da assimetria inter-hemisférica após a estimulação estaria relacionada às bases neurais dos ganhos funcionais induzidos pela reabilitação ${ }^{29}$. Assim, pode-se dizer que uma diminuição da assimetria seria indicativa de um retorno às condições homeostáticas por parte de mecanismos específicos subjacentes às alterações decorrentes de uma patologia distinta.

Como argumentado, o registro da atividade cortical possibilita maior entendimento acerca dos padrões neurais associados a processos cognitivos, sensoriais e motores. No processo de reabilitação, o conhecimento destes padrões neurais e dos mecanismos relacionados à integração sensório-motora e à construção do movimento voluntário é de extrema importância. Embora seja um aparelho importante para complementar e até potencializar a terapia, os efeitos do FES no organismo e mais especificamente na dinâmica cerebral, ainda são pouco conhecidos. Entretanto, a eletroestimulação tem demonstrado ser um protocolo confiável, uma vez que promove um recrutamento neuronal em áreas cerebrais seletas, na mesma quantidade em cada hemisfério, sendo independente de diferenças individuais na preparação motora e estratégias de execução do movimento. Os resultados do presente estudo demonstram que, assim como outras técnicas de estimulação elétrica, o FES possibilita uma redução da assimetria cortical, o que seria indicativo de uma normalização cerebral, estando de acordo com outros dados da literatura já mencionados.

\section{REFERÊNCIAS}

1. Ferrari M. Observing the observer: self-regulation in the observational learning of motor skills. Dev Rev 1996;16:203-240.

2. Plautz EJ, Milliken GW, Nudo RJ. Effects of repetitive motor training on movement representations in adult squirrel monkeys: role o use versus learning. Neurobiol Learn Mem 2000;74:27-55.

3. Weeks DL, Anderson LP. The interaction of observational learning with overt practice: effects on motor skill learning. Acta Psychol 2000;104: 259-271.
4. Vitenzon AS, Mironov EM, Petrushanskaya KA. Functional electrostimulation of muscles as a method for restoring motor functions. Neurosci Behav Physiol 2005;7: 709-714.

5. Veiga H, Deslandes A, Cagy M, Fiszman A, Piedade RA, Ribeiro P. Neurocortical electrical activity tomography in chronic schizophrenics. Arq Neuropsiquiatr 2003;61:712-717.

6. Schaul N. The fundamental neural mechanisms of electroencephalography. Eletroenceph Clin Neurophysiol 1998;106:101-107.

7. Gevins A, Smith ME, McEvoy LK, Leong H, Le J. Electroencephalographic imaging of higher brain function. Phil Trans R Soc Lond B Biol Sci 1999;354:1125-1133.

8. Oldfield R. The assessment and analysis of handedness: the Edinburgh inventory. Neuropsychologia 1971;9:97-113.

9. Jasper $H$. The ten-twenty electrode system of international federation. EEG Clin Neurophysiol 1958;10:371-375.

10. Miller A, Tomarken A. Task-dependent changes in frontal brain asymmetry: effects of incentive cues, outcome expectancies, and motor responses. Psychophysiology 2001;38:500-511.

11. Ginter J Jr, Blinowska KJ, Kaminski M, Durka PJ. Phase and amplitude analysis in time -frequency space- application to voluntary finger movement. J Neurosci Methods 2001;110:113-124.

12. Kandel E, Schwartz S, Jessel T. Principles of neuroscience. 4.Ed. New York: McGraw-Hill, 2000.

13. Fingelkurts AA, Rytsala H, Suominen K, Isometsa E, Kahkonen S. Composition of brain oscillations in ongoing EEG during major depression disorder. Neurosci Res 2006;56:133-144..

14. Chiarello C, Lombardino LJ, Kacinik NA, Otto R, Leonard CM. Neuroanatomical and behavioral asymmetry in an adult compensated dyslexic. Brain Lang 2006;98:169-181.

15. Penolazzi B, Spironelli C, Vio C, Angrilli A. Altered hemispheric asymmetry during word processing in dyslexic children: an event-related potential study. Neuroreport 2006;20;17:429-433.

16. Wiedemann G, Pauli P, Dengler W, Lutzenberger W, Birbaumer N, Buchkremer G. Frontal brain asymmetry as a biological substrate of emotions in patients with panic disorders. Arch Gen Psychiatry 1999;56: 78-84.

17. Smit DJ, Posthuma D, Boomsma DI, De Geus EJ. The relation between frontal EEG asymmetry and the risk for anxiety and depression. Biol Psycho 2007;74:26-33.

18. Pfurtscheller G, Stancak A, Neuper C. Post-movement beta synchronization. A correlate of an idling motor area? Electroencephalogr Clin Neurophysiol 1996;98:281-293.

19. Gross J, Pollok B, Dirks M, Timmermann L, Butz M, Schnitzler A. Taskdependent oscillations during unimanual and bimanual movements in the human primary motor cortex and SMA studied with magnetoencephalography. Neuroimage 2005;15;26:91-98.

20. Erickson KI, Colcombe SJ, Wadhwa R, et al. Training-induced plasticity in older adults: effects of training on hemispheric asymmetry. Neurobiol Aging 2007;28:272-283.

21. De Toffol B, Autret A, Markabi S, Roux S. Influence of lateralized sensorimotor and neuropsychological activities on electroencephalographic spectral power. Electroencephalogr Clin Neurophysiol 1990;75:200-206.

22. Tecchio F, Zappasodi F, Tombini $\mathrm{M}$, et al. Brain plasticity in recovery from stroke: an MEG assessment. Neuroimage 2006;32:1326-1334.

23. Nelles G, Spiekramann G, Jueptner M, Leonhardt G, Muller S, Gerhard, $\mathrm{H}$, Diener HC. Evolution of functional reorganization in hemiplegic stroke: a serial positron emission tomographic activation study. Ann. Neurol 1999;46:901-909.

24. Rossini PM, Tecchio F, Pizzella V, Lupoi D, Cassetta, E., Pasqualetti, P. Interhemispheric differences of sensory hand areas after monohemispheric stroke: MEG/MRI integrative study. Neuroimage 2001;14:474-485.

25. Oliviero A, Tecchio F, Zappasodi F, et al. Brain sensorimotor hand area functionality in acute stroke: insights from magnetoencephalography. Neuroimage 2004;23:542-550.

26. Nudo RJ, Wise BM, SiFuentes F, Milliken GW. Neural substrates for the effects of rehabilitative training on motor recovery after ischemic infarct. Science 1996;272:1791-1794.

27. Schallert T, Leasure JL, Kolb B. Experience-associated structural events, subependymal cellular proliferative activity, and functional recovery after injury to the central nervous system. J Cereb Blood Flow Metab 2000;20:1513-1528.

28. Meinzer M, Elbert T, Wienbruch C, Djundja D, Barthel G, Rockstroh B. Intensive language training enhances brain plasticity in chronic aphasia. BMC Biol 2004;2:20.

29. Ward NS. Mechanisms underlying recovery of motor function after stroke. Postgrad Med J 2005;81:510-514. 